\title{
Research Status and the Prospect of Nonsuicidal Self-Injury Behavior
}

\author{
Juan Chen ${ }^{1,2,3}$, He Chen ${ }^{1,2,3}$, Yuanyuan Jiang ${ }^{1,2,3}$ \& Lingkai Ma ${ }^{1,2,3}$ \\ ${ }^{1}$ Institute of Psychological Sciences, College of Education, Hangzhou Normal University, Hangzhou, China \\ ${ }^{2}$ Zhejiang Key Laboratory for Research in Assessment of Cognitive Impairments, Hangzhou, China \\ ${ }^{3}$ Center Cognition and Brain Disorder, Hangzhou Normal University, Hangzhou, China \\ Correspondence: He Chen, Institute of Psychological Sciences, College of Education, Hangzhou Normal \\ University, Zhejiang, China. Tel: 86-135-6790-2480.
}

Received: March 31, 2020

Accepted: April 10, 2020

Online Published: April 17, 2020

doi:10.20849/jed.v4i1.738

URL: https://doi.org/10.20849/jed.v4i1.738

\begin{abstract}
Nonsuicidal self-injury (NSSI), also referred to as self-injurious behavior (SIB), are different terms to describe behaviors where the demonstrable injury is self-inflicted. The behavior involves deliberate tissue damage that is usually performed without suicidal intent. The term self-mutilation is also sometimes used, although this phrase evokes connotations that some find worrisome, inaccurate, or offensive. The diagnostic study of this behavior is controversial, and it needs to be further discussed with the definition criteria of suicide and borderline personality disorder. The pathologic mechanism of nssi behavior is proposed by several theoretical models, including the functional model, developmental pathologic model, and integrative model. In the future research, the research on the psychological evaluation intervention of nssi behavior should be widened, the factors affecting nssi can be further classified, and the cross-cultural research on nssi behavior and the applicability of existing foreign research achievements in China will also become the focus of future research.
\end{abstract}

Keywords: nonsuicidal self-injury, self-injury, cross-cultural

\section{Summary of Nonsuicidal Self-Injury Behavior}

Nonsuicidal self-injury (NSSI) is different terms to describe behaviors, where the demonstrable injury is self-inflicted (Ross \& Heath, 2002; Whitlock, Eckenrode, \& Silverman, 2006). The behavior involves deliberate tissue damage that is usually performed without suicidal intent, and its related research has been paid more and more attention by researchers and clinical workers. Early studies defined nssi according to some abnormal behaviors, such as cutting/scratching with a sharp tool, burning with a lighter, hitting the head against the wall, etc., and the specific injury forms may exceed more than ten kinds (Yu Feng, 2008; Sinclair, Hawton, \& Gray, 2010; Ross \& Heath, 2002).

Nonsuicidal self-injury (NSSI) is a special psychopathological behavior, and its related research has been paid more and more attention by researchers and clinical workers. Early studies defined nssi according to some abnormal behaviors, such as cutting/scratching with a sharp tool, burning with a lighter, hitting the head against the wall, etc., and the specific injury forms may exceed a dozen (Helen Spandler 1996; Hao He, 2010; Yu Feng, 2008; Ying Zhao, 2006). Although nssi does not pose an immediate threat to the person's life or harm to others around him, it is highly insidious. It will cause physical injury to the parties, and the degree of injury also indicates that they are unable to deal with their bad emotions, stress, etc. (Briere \& Gil, 1998). It also indicates that these people are Shouting to themselves or calling for help from others through nssi, so they are the group that needs to attract professional attention and help. In the field of nssi behavior, domestic research has just started. Compared with some other pathological behaviors such as suicide, substance addiction, eating disorder, Internet addiction, etc., nssi behavior seems to have not entered the field of vision or been covered by other symbiotic problems, and has not attracted the attention of most researchers and practitioners in China (Gratz, 2006; Ross \& Heath, 2002). Only in recent years have researchers focused on nssi in non-clinical samples, especially adolescents. According to the research data at home and abroad, teenagers are the population with a high incidence of nssi, and there is an increasing trend Whitlock et al., 2006; Zoroglu et al., 2003). Briere (1998) showed that the incidence of nssi was $4 \%$ in the general population and $12 \%$ in the clinical population.

The diagnosis of nssi behavior has always been one of the controversial points in this study. The focus of the controversy is the relationship between nssi and suicide, nssi and borderline personality disorder. As for the 
theoretical paradigm of nssi behavior, from the existing research conclusions, researchers unanimously agree with the multidimensional functional model of nssi behavior. According to this model, the functional causes of nssi behavior of self-injurers are not single but varied. Different self-injurers, the causes of nssi are different; the same self-injury, each nssi cause can not be different. Among them, the explanation ratio of emotion management function is the largest. The research conclusion of zheng ying in China also supports the hypothesis that nssi has emotion management function, and the corresponding "experience avoidance model" is also proposed abroad (Ying Zhao, 2006). The occurrence of nssi is not the result of a single factor. Some studies have divided the influencing factors into two aspects: personal factors and environmental factors. Individual factors include gender, race, attachment style and emotion (Claes \& Vandereycken, 2007). Environmental factors can be divided into three aspects: family, school and society. In order to better understand the pathological mechanism of nssi behavior, it is necessary to explore the different factors that affect the occurrence of nssi behavior and the main groups from the perspective of etiology, link the theoretical model of nssi behavior, and further discuss the psychological evaluation and intervention of nssi behavior.

\section{The Concept of Nonsuicidal Self-Injury Behavior}

\subsection{Definition of Nssi Behavior}

The definition of nssi has always been a controversial point in this field. According to statistics, at least 33 terms have been used to refer to self-injurious behaviors (Brausch \& Gutierrez, 2009; Brown, Comtois, \& Linehan, 2002). Wherein, "seed self-harm (DSH)"; "The self - mutilation (SM)"; "The self-injury (SI)"; the four terms "para-suicide" are the most frequently used. In terms of the extension of the concept, SH covers the widest range, which can be understood as any behavior that directly or indirectly (such as smoking) causes harm to individuals. Behaviors that are accepted or even encouraged by culture are also included, such as body piercing, tattoos, and foot binding. DSH emphasizes the intentional nature of the behavior on the basis of SH. SI is further defined as socially unacceptable, non-suicidal, intentional and direct self-harming behavior in SH, and the damage to the body should be mild or moderate (Sutton, 2007). SM refers to more serious SI behavior, such as limb amputation and eye gouging. Although researchers have been unable to form a unified definition of nssi behavior, comprehensive literature in recent years can be summarized as :1) no suicide motive, which reflects the essential difference between nssi behavior and suicide behavior; 2) intentionality, that is to say, the nssi behavior taken by individuals should be conscious rather than automatic or unconscious; 3 ) the degree of damage, the behavior of self-injury should cause slight or moderate physical injury, while the major and fatal injury, such as eye gouging, should be excluded 4) repetition, self-injury behavior should be repeated, while fatal injury behavior is often difficult to repeat; 5) not recognized by the society, self-harming behavior is not accepted and recognized by the society. According to the definition of root Gratz (2001), self-injury behavior refers to the deliberate and repeated modification or injury of an individual's own body tissue without suicidal intent, but the behavior is not fatal or less fatal.

\subsection{Identification of Nssi Behavior}

\subsubsection{Nssi and Suicide}

In recent years, researchers in the field of nssi behavior believe that the above confusion of terms should not exist, because the motives behind nssi behavior and suicide are not the same. Although Favazza (1996) found that $28 \% \sim 41 \%$ of self-injurers reported having suicidal thoughts when performing nssi; Stanley et al. (1992) found that $55 \% \sim 85 \%$ of individuals who performed nssi had at least one suicide attempt. Stanley et al. (2001) studied 53 clinical patients with BPD and found that there was no significant difference in the number of suicidal ideation and attempted suicide between nssi and non-self-injurers. Current research suggests that suicidal people have an intention to die, while self-injurers have no intention to die. Another piece of evidence distinguishes nssi behavior from suicide, that is, nssi behavior has a low fatality rate.

According to the "National Center for Injury Prevention and Control" (National Center of Injury Prevention and Control), according to a survey in 2005 under the age of 24, self-injury population and total self-injury mortality rates were $0.4 \%$ and $0.6 \%$ respectively, although the mortality rate is higher than the general population, on the relationship between the two actions, this indicates that the behavior of two correlation is not strong (Guangrong Jiang, lixia Yu, Yu, Z. Y. F., 2011). From the perspective of empirical research, the nssi group with or without suicidal intention showed significant differences in depression, anxiety, self-esteem, social support, sense of despair, reasons for nssi/suicide, and coping ability.

\subsubsection{Nssi Behavior and Borderline Personality Disorder (BPD)}

There is a close relationship between Borderline Personality Disorder (BPD) and self-injury behavior. The 
researchers' attention to self-injury behavior is starts from the study of clinical samples of BPD, and DSM-IV regards self-injury as an indicator of BPD diagnosis (Meghan \& Daniel, 2015; Zheng et al., 2019). There is a common core problem behind the Boundary Personality Disorder (BPD) and self-injury behavior: the disorder of emotional management of individuals. Emotional management disorder are the core problems of self-injured individuals, which are manifested in the fragility of emotional feelings (emotions are easy to arouse, high intensity and difficult to calm down) and lack of emotional management capabilities.

However, self-injury behavior is different from Borderline Personality Disorder (BPD). A lot of studies have confirmed that self-injury behavior exists not only in BPD patients, but also in a large number of ordinary people (especially adolescents) (Claes et al., 2009). In addition, self-injury behavior is not only manifested in borderline personality disorder (BPD), but also in other disorders. For example, the 1999 McLean Study of Adult Development funded by the National Institute of Mental Health (NIMH) conducted a 10-year follow-up study of 299 people who met the symptoms of BPD $(\mathrm{N}=264)$. The incidence of self-injury has dropped from $81 \%$ to $26 \%$, and the proportion of parties recovering from BPD symptoms or having mild symptoms after about 10 years $(\mathrm{N}=249)$ is approximately $50 \%$. The two are different obstacles.

\section{The Theoretical Model of Self-Injure Behavior}

\subsection{Functional Research}

The functional model is designed to explore the underlying motivations and causes of self-injury behaviors. It explores all the factors related to self-injury behavior and explores the relationship among these factors. To note that, the functional model not only care about the factors during the self-injury, but also cares about the factors before and after self-injure. This model is originally derived from Iwata and Carr's motivational theory of innocent self-harm behavior and the experimental method of "functional analysis" and aims to meet the needs of clinical intervention (Ross \& Heath, 2002). Moreover, the functional model can provide a set of theoretically supported, operable, and testable intervention ideas for clinical use, and have gained empirical support for clinical effectiveness (Lloyd \& Hope, 1997). However, there are also some shortcomings of functional model. Some behaviors studied by functional models are overlapped. For example, the action of self-punishment is accompanied by negative emotions such as anxiety, frustration, and anger. It is hard to define whether the function of self-injury is self-punishment or emotional management. It seems that self-punishment and emotional management do not belong to different behavior categories, but more like the results that evaluate the same phenomenon in different aspects.

Due to the verifiability of theory and the effectiveness of the clinical intervention, the functional research of nssi has attracted the attention of a large number of researchers, and many functional models have been extracted. Suyemoto (1998) integrated six types of models from the four aspects of the environment, drive, emotional management and interpersonal function: environmental model, suicidal model, sexual model, emotional management model, separation model and boundary model. Messer and Fremouw (2008), from the literature of 1980-2008, summarized the models with high frequency into seven categories: sexual/abnormal model, depersonalization model, interpersonal/system model, suicide model, psychological/biological model, emotional management model and behavioral/environmental model. Klonsky (2007) concluded from 18 reports that there are seven functions that have been confirmed by research: emotional management, fighting against the sense of separation, fighting against suicide, restoring the boundary between oneself and others, interpersonal influence, self-punishment and feeling seeking. Among them, the function of emotion management was consistently supported by all studies, and the functions of self-punishment (11/18), coping with the sense of separation (10/18) and interpersonal influence (10/18) were also verified by most studies.

\subsection{Integrated Model}

\subsubsection{Two-Dimensional Model}

The two-dimensional model is an integrated model (Townsend \& Altman, 2001). This model divides self-injury into four categories from two dimentions of "individual-interpersonal" and "positive reinforcement-negative reinforcement", including positive individual reinforcement, negative individual reinforcement, positive interpersonal reinforcement and negative interpersonal reinforcement. The empirical research supports the Two-dimensional Model to a certain extent, but not completely consistent with the theoretical hypothesis. The study shows that the relative strengths among four functional factors are not equal (Walsh, 2006). Therefore, Two-dimensional Model may mislead researchers and practitioners to treat different factors equally. The two-dimensional model has a particularly good effect in the introduction and evaluation of self-injury behavior. 


\subsubsection{Experiential Avoidance Model}

Experiential Avoidance Model (EAM) is a model mainly based on behaviorism, which believes the self-injury is maintained and strengthened through the process of avoidance and effective negative reinforcement. In order to get rid of the unpleasant emotion, the individual is likely to perform self-injury behavior under the mutual stimulation of various factors, and this behavior will help the individual get relieved from instant gratification. In other words, the connection between this unpleasant emotional stimulus and self-injury behavior forms a negative reinforcement, making self-injury behavior an automated escape response (Townsend \& Altman, 2001). Although the model believes that self-injury is primarily an emotional avoidance behavior, it can also help individuals avoid unwanted thoughts, memories, physical feelings, or other disgusting inner experiences. The EAM proposes several important factors that is, high emotional intensity, low tolerance, lack of emotional regulation strategies, and inability to effectively deal with emotional arousal. The main contribution of EAM is to refine the process of self-injury behavior and explain the vicious circle of self-injury behavior. However, it still requires further research to verify if the model has cross-cultural validity (Weierich, \& Nock, 2008).

\subsubsection{The Model of Developmental Psychopathology}

The Model of Developmental Psychopathology discusses how the trauma experience of childhood influences self-injure behavior. The model believes that individuals should develop five psychological abilities in the process of active adaptation: motivation, attitude, tools, emotions, and interpersonal. However, the traumatic experience of childhood will hinder the development of these abilities (Sandman \& Hetrick, 1995), which causes a lack of psychological ability. As a compensatory regulatory strategy, individuals will conduct self-injury behavior to compensate for their psychological ability in the process of personal growth. Therefore, the development model can be regarded as a model of self-injury etiology, which emphasizes the role of individuals' internal factors rather than the role of environmental factors. The contribution of severe character pathology, aggression, impulsivity, anxiety, and anger to self-mutilation and provide preliminary support for the hypothesis of underlying serotonergic dysfunction facilitating self-mutilation.

Both the two-dimensional model and EAM are functional models, based on the behaviorist reinforcement theory, which emphasizes the "external" factors of individuals (such as the ring). The role of circumstance, stimulus, behavioral outcome. The development model can be regarded as the etiological model of nssi, mainly based on the object relations theory, emphasizing the role of individual "internal" factors. The five abilities in the model belong to psychological ability, and the direct cause of nssi is the lack of psychological ability, rather than the role of environmental factors. The development model expounds on the developmental path of nssi caused by the early traumatic experience, which has strong logical positivism.

\subsubsection{Integrated Theoretical Model}

The integrated theoretical model is a comprehensive model proposed by Nock (2009), which includes biological, psychological, and social factors. He describes self-injury behavior from three aspects: the function of self-injury, risky factors and mechanism of self-injury, and the induce factors of self-injury. (a) The function of self-injury behaviors. Nock believes that self-injury is not only a coping mechanism for emotional and cognitive management, but also a way to communicate with or influence others; (b) Risky factors and mechanism of self-injury behaviors. The combined effects of genetic factors, early experience, and parenting styles will cause problems in the emotional management and interpersonal communication of the individual, which further led to self-injury behaviors; (c)The induce factors of self-injury behaviors. For the induce factors of self-injury behavior, Nock proposed six hypothetical variables: social learning, self-punishment, social signals, pragmatism, lack of pain, and potential identification.

The integrated theoretical model is the most comprehensive of the four models. It is not confined to any particular school of theory, but takes into account biology, psychological and social factors provide a whole way for researchers to understand the development and occurrence of nssi. The integrated theoretical model is more suitable as a theoretical framework to understand nssi. Due to the numerous factors and variables involved, it is difficult to select and control in specific research, which brings difficulties to the verification of the model.

Although the above theoretical models have different interpretations of self-injury, there are still some common points. First, they all attach great importance to the impact of the individual unhealthy family environment on self-injury behavior, especially early traumatic experiences such as abuse, neglect, etc. Second, these models emphasize the impact of individual emotions on self-injury behavior, mainly including emotional expression disorders and emotional regulation disorders. Finally, these models stress that the unhealthy family environment at the early stage may affect people's ability to develop effective emotional management and regulation, which may lead to self-injury behaviors. 


\section{Influencing Factors of Self-Injurious Behavior}

\subsection{Early Traumatic Experience}

According to the existing literature on self-injury research, the early traumatic experiences mainly including childhood abuse (physical, sexual and emotional abuse), childhood neglect, early separation and loss. Earlier studies did not classify each traumatic experience. Abuse can be divided into sexual, physical, and emotional abuse; neglect can be divided into physical and emotional neglect. Recent studies have mostly examined the relationship between different subtypes and self-injury. Although the significance levels of different research are mixed, the correlation between subtypes and self-injury is basically stable at about 0.2 to 0.3 (Yu, Jiang \&Wu, 2011). Klonsky and Moyer (2008) use meta-analysis to confirm the relationship between sexual abuse and self-injury behavior. The results show that the two variables are related to 0.23 . Overall, sexual abuse does not explain more than 5\% of self-injury behavior. Klonsky and Moyer (2008) believe that childhood sexual abuse is unlikely to have a major effect on postnatal self-injury behavior (Favazza, 1998). Finally, when researchers put several potential risk factors (including physical abuse, emotional abuse, sexual abuse, and loss of separation) into the regression equation to predict self-injury behavior, only childhood sexual abuse was significantly predicted. The reason why these current research results are not consistent may be due to the large differences between samples, the interaction between congenital factors and acquired factors, etc., or the use of different measurement tools. For different measurement tools, the nature of the selected sample is different, which may cause more influence of additional variables. From the nature of the sample, there are freely recruited social personnel (Glassman et al., 2007), a group of college students (Gratz, Conrad, \& Roemer, 2002), and residents of mental rehabilitation centers (Weierich \& Nock, 2008), bottom income groups (Yates et al., 2008), etc. (Gerbing, Ahadi \& Patton, 1987; Glassman et al., 2007).

\subsection{Individual Susceptibility}

\subsubsection{Emotional Management Disorder}

The self-injury behavior parties use self-injury to vent and adjust their emotions, which may be their lack of adaptive emotion regulation strategies. This type of individual has an emotional vulnerability in terms of emotions. Linehan (1993) pointed out that the main manifestations of emotional susceptibility of self-injured individuals are: high emotional intensity and a low tolerance for emotions. Chapman et al. (2006) believe that the emotional management of self-injured patients is manifested in three aspects: inability to express emotions, difficulty in regulating emotions, and high emotional intensity. Emotional incapacity called Alexithymia, it is one of the cognitive-affective disorder that affects individual emotional experience and expression. Emotional regulation refers to an individual's awareness, understanding, acceptance of his own emotional experience, and flexible use of strategies to make appropriate behaviors (Feng, 2008). Similarly, difficulty in emotional regulation refers to a lack of any of the above abilities. A great many empirical studies have shown that emotional regulation difficulties can effectively predict self-injury behaviors (Brausch et al., 2009). KLaus (2004) proposed the BPD neurobehavioral model, focusing on the effects of emotional disorders on poor behaviors. Few studies have directly examined the direct relationship between emotional expression and emotional regulation.

\subsubsection{Innate Personality or Temperament}

Studies have found that the time interval between self-injury and the formal implementation of self-injury is usually less than five minutes. Impulsivity is a personality trait with a strong biological basis, mainly manifested as the tendency of individuals to react quickly and unplanned to internal or external stimuli without considering the negative consequences to themselves or others (Gratz et al., 2001; Gratz et al., 2007). Recent studies have shown that there is still no consensus among researchers on the concept and structure of impulsivity. Since measurement tools rely on specific theoretical framework (Gratz et al., 2002), the inconsistency of theoretical definition also leads to the diversification of impulse measurement tools (Hastings, Noone, 2005). During the measurement, since self-reporting is affected by individual subjective factors, researchers usually recommend that the self-reporting be combined with objective indicators such as behavioral science, neurobiology and neuropsychology for comprehensive evaluation (Brausch\&Gutierrez, 2009).Though impulsivity is one of the important influencing factors of self-injury, the conclusions of impulsivity research on self-injured people are inconsistent. The first is the conflict between the findings. Herpertz et al. (1997) found that there was no significant difference in impulsivity between the self-injury group and the control group (Hayes et al., 1996). Glenn and Klonsky (2010) used the UPPS impulsivity scale (Brausch et al., 2009) and the stop-signal task to explore the relationship between impulsivity and self-injury. The results confirmed that the UPPS measurement results in the self-injury group were significantly higher than those in the control group, while two groups 
performed consistently in the stop-signal task (Yu, Jiang\&Wu, 2011).

\subsubsection{Biological Factors}

Some evidence from animal experiments and neurophysiology / biology also reminded researchers to pay attention to the role of genetic factors. In the early years, researchers found the same self-injury phenomenon in primates as humans (Jiang et al., 2011). Clinical studies have also found that altering biological substances in the body (cycloprofenol, a narcotic antagonist) can provide effective interventions for self-injury (Herpertz et al., 1997). These provide evidence for possible biological effects. The biological factors considered to be related to self-injury include endogenous opioid peptides, serotonin, dopaminergic system, hypothalamic-pituitary-adrenal axis, etc. Among them, endogenous opioid peptides are considered to play a key role. The reason is that it may be closely related to a pathological experience of self-injury-painlessness (Jiang et al., 2011).

\section{Psychological Evaluation and Intervention of Nonsuicidal Self-Injury Behavior}

\subsection{Evaluation of Nonsuicidal Self-Injury Behavior}

\subsubsection{Behavioral Motivation}

In order to assess whether a person with suicidal behavior is suicidal, it is first necessary to understand their motivation. It is easy to distinguish between suicidal behavior and suicide in theory, but it is difficult to distinguish in actual operation clearly. In recent studies, there have been many suicide attempts by suicidal parties. If the parties have suicidal intentions, they should be taken seriously immediately.

\subsubsection{Mental Disorders}

There is a symbiotic relationship between self-injury behavior and borderline personality disorder (BPD), eating disorders, depression, anxiety, drug abuse, post-traumatic stress disorder (PTSD), and separation disorders (Brown, Comtois\& Linehan, 2002). If the client is found to have obvious symptoms of mental/psychological disorder and meets the diagnostic criteria, the counselor should first consider appropriate medication. There is no research evidence that drug treatment can effectively reduce self-injury behavior, but drug treatment can effectively reduce the mental symptoms related to self-injury, such as anxiety, depression, BPD, etc. (Linehan et al., 2006).

\subsubsection{The History of Self-Injury}

The history of self-injury is mainly aimed to understand the basic information related to self-injury, including the mode of self-injury, the frequency of self-injury, the location of the self-injury, the degree of injury, the time of the first self-injury, the time of the most recent self-injury, etc. Whether there are other pathological behaviors such as drug abuse, alcoholism, and eating disorders. Studies have concluded that the more methods used during self-injury, the greater the likelihood of attempted suicide (Gratz, 2001; Lloyd et al., 1997).

\subsubsection{Functional Evaluation}

Functional evaluation was originally produced in response to the need for clinical intervention. Zheng Ying (2006) interviewed Chinese self-injury adolescents and found that self-management and control of others are the two main causes of self-injury. The main information that needs to be collected to assess the self-injury function includes: (a) The situation that induces self-injury, that is, what happened before the injury, and which important others are involved in these events. (b) The feelings and experiences of the parties. (c) The cognitive beliefs of the parties, namely the thoughts of the parties. (d) The result of self-injurious behavior. Based on the information in these four aspects, follow-up treatment can know how to cause and maintain the occurrence of self-injury behavior (Claes \& Vandereycken, 2007).

\subsection{Common Evaluation Tools}

\subsubsection{Functional Assessment of Self-Mutilation (FASM)}

Mainly assess the mode, frequency and function of self-injury. The questionnaire consists of two parts. The first part understands the basic situation of self-injury in the past one year, including the mode of self-injury, the frequency of occurrence of self-injury, the severity, the status of treatment, and the duration of self-injury, the age of the injure, whether it was affected by drugs and alcohol at the time of the injure, the degree of pain in the body, and whether it was an attempted suicide. The second part understands the motivation for self-injury. FASM has better reliability and validity in adolescent samples.

\subsubsection{Deliberate Self-Injury Inventory (DSHI)}

Seventeen items were used to measure the frequency, severity, duration, and type of self-injury. Gratz conducts research with a sample of 150 college students, cronbach's alpha was 0.82 , Retest reliability of 0.92 in two to 
three weeks. DSHI has ideal structural validity, convergence validity, discriminative validity, and predictive validity (Muehlenkamp \& Gutierrez).

\subsubsection{The Suicide Attempt Self-Injury Interview (SASII)}

SASII is a structured interview tool based on multiple groups of clinical samples. Professional trainees judge whether a certain suicidal behavior is a non-suicide suicide, a suicide attempt with inner conflict, or a clear attempted suicide. The SASII has 6 items (suicidal ideation, interpersonal influence, emotional release, suicidal expression, lethality and possibility of being rescued). Cronbach's alpha was 0.87-0.98; the measurement results are highly consistent with the patient's actual physical condition, the results reported by the consultant, the client's diary and medical records. The identification rate of self-injury patients in clinical treatment reached $83 \%$, and there were no false positives.

\subsubsection{Self-Injury Thought Sand Behavior Interview (SITBI)}

SITBI is a structural interview tool, it has 169 items. It is used to evaluate the frequency and characteristics of thoughts, behaviors related to suicidal ideation, suicide plan, suicidal attitude, attempted suicide and non-suicide suicide. Measurements from a sample of 94 non-hospitalized adolescents showed that the cohen's kappa was 0.99. Except for suicide attitude $(\mathrm{k}=0.25)$, the other 4 types of behaviors have a 6-month test result with cohen's kappa of $0.7 \sim 1.0$; the cohen's kappa of SITBI and other suicidal ideas, attempted suicide and non-suicide suicide measures $0.54-0.87$.

\subsubsection{Middle School Student Behavior Questionnaire}

The self-assessment scale for the parties was based on the FASM and was revised through the results of open interviews with more than 30 self-injury adolescents in Wuhan's school. Participants rated the extent to which statements described themselves on a 4-point scale ranging from 1 to 5, including 20 self-injury methods, 38 self-injury functions and self-injury history. The test results of 1,283 ordinary junior high school students show that the functions of self-injury are divided into four dimensions: self-management, interpersonal control, strength display and stimulation seeking. Cronbach's alpha was 0.92 . The one-month retest reliability of the self-injury mode and the functional subscale were 0.79 and 0.83 .

\subsection{Treatment of Self-Injury Behavior}

\subsubsection{Therapeutic Relationship}

A good therapeutic relationship is a basis for the evaluation and successful treatment of self-injury behaviors (Claes \& Vandereycken,2007), and some studies have found that consultants' attitudes of low attention, calmness, and non-evaluation to visitors are considered to be the most helpful in assessing and treating self-injury behaviors (Nafisi \& Stanley). The excessive attention and tension of the consultant may become a secondary benefit and strengthen the occurrence of self- injury behavior. If it is an evaluation attitude, it may make some sensitive parties' conflict.

\subsubsection{Target of Treatment}

Klonsky (2007) summarized seven confirmed functions from 18 research reports on self-injury functions. Among them, the emotional management function has been supported by all researches. Therefore, the goal of treating self-injury behaviors is to acquire emotional management skills. So whether emotion management manages emotions or management behavior has always been puzzling. Managing emotions is equivalent to controlling or reducing them. In clinical terms, negative emotions are prone to problems and problems. However, from the perspective of individual adaptation or development, experiencing negative emotions under normal circumstances is a normal and healthy response. From a clinical perspective, normal and abnormal emotions are relative, relative to specific circumstances. Therefore, individuals can control their behavior if the parties experience negative emotions. Gratz and Roemer (2007) summarized the ability of emotion management into four aspects. (a) Aware, understand and accept your emotions; (b) Can inhibit impulsive behavior or engage in other target-oriented cathartic behaviors when experiencing negative emotional experiences; (c) Can flexibly adjust the intensity and duration of emotional reactions in different situations, instead of completely eliminating emotions; (d) Can regard negative emotions in the pursuit of meaningful activities as a necessity of life (Nock, 2009).

\subsubsection{Treatment Method}

There are few empirical studies on the treatment of self-injury behavior, and the majority come from the treatment evaluation of BPD patients. Among them, most empirical tests are problem-solving treatment and dialectical behavior therapy (DBT). The meta-analysis found that problem-solving therapy significantly 
improved the symptoms of comorbidities such as depression and despair among the self-injuryd parties (Herpertz et al., 1997). There is no statistically significant difference between PST and other therapies. DBT is a behavioral therapy derived from BPD. It is especially effective for individuals with suicidal behavior and severe dysfunction (BPD) (Nock et al., 2009). For clinicians, the key to effective treatment is not what treatment technology or procedure is used, and the self-injury group is highly heterogeneous, and it is difficult to develop a standardized treatment procedure that is effective for all parties. The key to effective treatment is that the consultant has a thorough understanding of the phenomenon and nature of self-injury, functional characteristics, and the focus of treatment, and a deep understanding of the causes of self-injury by specific parties.

\section{Summary and Outlook}

\subsection{Summary Nonsuicidal Self-Injury Behavior for the Different Aspects}

\subsubsection{Classification Research}

The definition of self-injury behavior has been controversial. Researchers have conceived that self-injury may contain inherently heterogeneous components, and it is precisely these heterogeneous components that have led to conflicting research conclusions. To deal with this possible heterogeneity, a classification study is a logical answer. Ross and Mckay (1979) tried to classify self-injury behaviors into nine types of cutting, biting, and burning according to the type of injury; Simeon (1995) classified them into formal, severe, compulsive and impulsive self-injury based on experience. In future studies, a deeper understanding of self-injury behaviors will make people's understanding of self-injury behaviors clearer, and further research on the analysis of specific types of self-injury behaviors (Whitlock et al., 2006).

\subsubsection{Interdisciplinary Research}

As a kind of psychopathological behavior, the research on self-injury is mostly concentrated in the field of clinical psychology. The research methods mainly use introspective self-reporting and experimental methods, and self-reporting questionnaires and interview methods are mainly used (Yates et al., 2008). On the other hand, self-injury behavior can also be found in biochemical research, neurophysiological research or zoology, and it has found some empirical research findings, which plays an important role in the concept and pathological mechanism of self-injury behavior. Therefore, interdisciplinary research has provided research evidence for the in-depth mechanism of self- injury behavior and the biological influence factors. The evidence from these studies may provide a new method for our research on the factors affecting self-injury and classification.

\subsubsection{Research Objects}

At present, most of the domestic researches focusing on self-injury behavior focuses on adolescent groups. Feng Yu and Zheng Ying (2006) have related research evidence on this research object. In future research, self-injury behavior can be expanded. To the elderly. Due to the phenomenon of "empty nest elderly," the self-injury behavior of some elderly may directly lead to suicide.

\subsubsection{Cross-Cultural Research}

Western attention to self-injury has been around for a century. As early as 1880 , self-injury behavior was noted in clinical samples of borderline personality disorder (BPD) abroad, and it was not distinguished from other behavioral problems of mental disorders as an independent category at that time. In the past decade, related research has increased dramatically. In the early years, he mainly described the psychological or behavioral characteristics of self-injury individuals. Later, he paid more attention to in-depth interpretation of self-injury, such as influencing factors, functions, etiology and pathology. Although there has been considerable research, due to the complexity of self-injury behavior, researchers have not reached consensus on many issues (Brausch $\&$ Gutierrez, 2009). As early as the 1980s, China introduced the "self-injury syndrome". Since then, the few literatures are mainly case reports of clinicians. In recent years, some empirical studies have appeared, and some valuable research findings have been drawn. Based on the differences between Chinese and Western cultures, whether foreign research results are adaptive in China is also a concern for future research. Chinese teenagers are more likely to face conflicts between external requirements and individual internal needs, and academic pressures such as adolescence may It is also one of the reasons for the high incidence of self-injury behavior in this group, and the specific results need further investigation and research to prove.

\subsection{Contribution and Limitation of the Study}

There were a few limitations to our study. First, the factors that influence self-injurious behavior were not explored sufficiently. Although earlier traumatic experiences also included the environmental factors such as family and school environment, the discussion on how these factors influence self-injurious behavior was not 
specific enough. Future researches can specifically explore the influence of factors such as living or working environment on self-injurious behavior. Second, the current assessment methods for self-injurious behaviors were mainly concentrated in the field of questionnaires. It is not clear whether the other indicators such as physiological indicators can detect self-injurious behaviors. Future researches can explore and list the neural mechanism and their detection indicators of self-injurious behavior.

Despite these limitations, the present study demonstrated how factors such as early traumatic experience and individual susceptibility influence the occurrence of self-injurious behavior. Furthermore, by introducing different models of self-injurious behavior, this study discussed self-injurious behavior from the perspective of pathogenicity and listed the progress of existing research on the psychological treatment of self-injurious behavior.

Above all, the current study introduced the definition of self-injurious behavior and systematically sorts out its influencing factors, models, and intervention methods. This information could help people familiar with the researches in the field of self-injury behavior more quickly and may provide some useful information for them to find new directions.

\section{Acknowledgement}

Juan Chen and He Chen were supported by the provincial advantage displine construction, talent cultivation, and research innovation from the college of Education, Hangzhou Normal University.

\section{References}

Brausch, A. M., \& Gutierrez, P. M. (2009). Differences in non-suicidal self-injury and suicide attempts in adolescents. Journal of Youth and Adolescence, 39(3), 233-242. https://doi.org/10.1007/s10964-009-9482-0

Briere, J. N., \& Gil, E. (1998). Self-mutilation in clinical and general population samples: prevalence, correlates and functions. American Journal of Orthopsychiatry, 68(4), 609-620. https://doi.org/10.1037/h0080369

Brown, M. Z., Comtois, K. A., \& Linehan, M. M. (2002). Reasons for suicide attempts and nonsuicidal self-injury in women with borderline personality disorder. Journal of Abnormal Psychology, 111(1), 198-202. https://doi.org/10.1037/0021-843X.111.1.198

Carr, J., LeBlanc, L., \& Love, J. (2008). Cognitive behavior therapy: Applying empirically supported techniques in your practice (pp. 211-221). Experimental functional analysis of problem behavior.

Chapman, A. L., Gratz, K. L., \& Brown, M. Z. (2006). Solving the puzzle of deliberate self-harm: the experiential avoidance model. Behaviour Research and Therapy, 44(3), 371-394. https://doi.org/10.1016/j.brat.2005.03.005

Claes, L., \& Vandereycken, W. (2007). Self-injurious behavior: Differential diagnosis and functional $\begin{array}{llll}\text { differentiation. } & \text { Comprehensive } & \text { Psychiatry, } & \text { 48(2), }\end{array}$ https://doi.org/10.1016/j.comppsych.2006.10.009

Claes, L., Muehlenkamp, J., Vandereycken, W., Hamelinck, L., Martens, H., \& Claes, S. (2010). Comparison of non-suicidal self-injurious behavior and suicide attempts in patients admitted to a psychiatric crisis unit. Personality and Individual Differences, 48(1), 83-87. https://doi.org/10.1016/j.paid.2009.09.001

Evenden, J. L. (1999). Varieties of impulsivity. Psychopharmacology, 146(4), 348-361. https://doi.org/10.1007/PL00005481

Favazza, A. R. (1998). The coming of age of self-mutilation. Journal of Nervous and Mental Disease, 186, 259-268. https://doi.org/10.1097/00005053-199805000-00001

Feng, Y. (2008). The relationship between adolescent self-injurious behavior and individual emotional factors and family environment factors. Master's Thesis, Central China normal university.

Gerbing, D. W., Ahadi, S. A., \& Patton, J. H. (1987). Toward a conceptualization of impulsivity: Components across the behavioral and self-report domains. Multivariate Behavioral Research, 22(3), 357-379. https://doi.org/10.1207/s15327906mbr2203_6

Glassman, L. H., Weierich, M. R., Hooley, J. M., Deliberto, T. L., \& Nock, M. K. (2007). Child maltreatment, non-suicidal self-injury, and the mediating role of self-criticism. Behaviour Research and Therapy, 45(10), 2483-2490. https://doi.org/10.1016/j.brat.2007.04.002

Glenn, C. R., \& Klonsky, E. D. (2010). A multimethod analysis of impulsivity in nonsuicidal self-injury. Personality Disorders: Theory, Research, and Treatment, 1(1), 67-75. https://doi.org/10.1037/a0017427 
Gratz, K. L. (2001). Measurement of deliberate self-harm: Preliminary data on the Deliberate Self-Harm Inventory. Journal of Psychopathology and Behavioral Assessment, 23(4), 253-263. https://doi.org/10.1023/A:1012779403943

Gratz, K. L., \& Chapman, A. L. (2007). The role of emotional responding and childhood maltreatment in the development and maintenance of deliberate self-harm among male undergraduates. Psychology of Men \& Masculinity, 8(1), 1-14. https://doi.org/10.1037/1524-9220.8.1.1

Gratz, K. L., Conrad, S. D., \& Roemer, L. (2002). Risk factors for deliberate self-harm among college students. American Journal of Orthopsychiatry, 72(1), 128-140. https://doi.org/10.1037/0002-9432.72.1.128

Hastings, R. P., \& Noone, S. J. (2005). Self-injurious behavior and functional analysis: Ethics and evidence. Education and Training in Developmental Disabilities, 40(4), 335-342.

Hayes, S. C., Wilson, K. G., Gifford, E. V., Follette, V. M., \& Strosahl, K. (1996). Experiential avoidance and behavioral disorders: A functional dimensional approach to diagnosis and treatment. Journal of Consulting and Clinical Psychology, 64(6), 1152-1168. https://doi.org/10.1037/0022-006X.64.6.1152

He, H. (2010). The relationship between physiological arousal, stress tolerance and social skills in adolescents with nonsuicidal self-injury. Master's Thesis, Central China normal university.

Herpertz, S., Sass, H., \& Favazza, A. (1997). Impulsivity in self-mutilative behavior: Psychometric and biological findings. Journal of Psychiatric Research, 31(4), 451-465. https://doi.org/10.1016/S0022-3956(97)00004-6

Jiang, G. R., Yu, L. X., Zheng, Y., Feng, Y., \& Ling, X. (2011). The Current Status, Problems and Recommendations on Non-Suicidal Self-Injury in China. Advances in Psychological Science, 19(6), 861-873.

Klonsky, E. D., \& Moyer, A. (2008). Childhood sexual abuse and non-suicidal self-injury: meta-analysis. British Journal of Psychiatry, 192(3), 166-170. https://doi.org/10.1192/bjp.bp.106.030650

Linehan, M. M., Comtois, K. A., \& Murray, A. M. (2006). Two-year randomized controlled trial and follow-up of dialectical behavior therapy vs therapy by experts for suicidal behaviors and borderline personality disorder. Arch Gen Psychiatry, 63(7), 757-766. https://doi.org/10.1001/archpsyc.63.7.757

Ling, X. (2009). ERPs study on impulse control in nssi adolescents. Master's Thesis, Central China normal university.

Lloyd, E. E., Kelley, M. L., \& Hope, T. (1997). Self-mutilation in a community sample of adolescents: Descriptive characteristics and provisional prevalence rates. Poster presented at the annual meeting of the Society for Behavioral Medicine. New Orleans. LA.

Meghan, A. M., \& Daniel, L. S. (2015). Dsm-5: diagnostic and statistical manual of mental disorders.

Moeller, F. G., Barratt, E. S., Dougherty, D. M., Schmitz, J. M., \& Swann, A. C. (2001). Psychiatric aspects of $\begin{array}{llll}\text { impulsivity. American Journal of Psychiatry, } & 158(11), & 1783-1793 .\end{array}$ https://doi.org/10.1176/appi.ajp.158.11.1783

Muehlenkamp, J. J. (2005). Self-Injurious Behavior as a Separate Clinical Syndrome. American Journal of Orthopsychiatry, 75(2), 324-333. https://doi.org/10.1037/0002-9432.75.2.324

Muehlenkamp, J. J., \& Gutierrez, P. M. (2007). Risk for Suicide Attempts Among Adolescents Who Engage in Non-Suicidal Self-Injury. Archives of Suicide Research, 11(1), 69-82. https://doi.org/10.1080/13811110600992902

Nafisi, N., \& Stanley, B. (2007). Developing and maintaining the therapeutic alliance with self-injuring patients. Journal Clin Psychol, 63(11), 1069-1079. https://doi.org/10.1002/jclp.20414

Nock, M. K. (2009). Why do people hurt themselves? New insights into the nature and functions of self-injury. $\begin{array}{lllll}\text { Current Directions in Psychological } & \text { Science, } & 18(2), & 78-83 .\end{array}$ https://doi.org/10.1111/j.1467-8721.2009.01613.x

Nock, M. K., \& Prinstein, M. J. (2004). A functional approach to the assessment of self-mutilative behavior. $\begin{array}{lllll}\text { Journal of Consulting and Clinical Psychology, } & \text { 72(5), }\end{array}$ https://doi.org/10.1037/0022-006X.72.5.885

Nock, M. K., \& Prinstein, M. J. (2005). Contextual features and behavioral functions of self-mutilation among adolescents. 
https://doi.org/10.1037/0021-843X.114.1.140

Nock, M. K., Joiner, T. E., Gordon, K. H., Lloyd-Richardson, E., \& Prinstein, M. J. (2006). Non-suicidal self-injury among adolescents: Diagnostic correlates and relation to suicide attempts. Psychiatry Research, 144(1), 65-72. https://doi.org/10.1016/j.psychres.2006.05.010

Ownsend, E., Hawton, K., \& Altman, D. G. (2001). The efficacy of problem-solving treatments after deliberate self-harm: Meta-analysis of randomized controlled trials with respect to depression, hopelessness and improvement in problems. Psychol Medicine, 31(6), 979-988. https://doi.org/10.1017/S0033291701004238

Ross, S., \& Heath, N. (2002). A study of the frequency of self-mutilation in a community sample of adolescents. Journal of Youth and Adolescence, 31(1), 67-77. https://doi.org/10.1023/A:1014089117419

Sandman, C. A., \& Hetrick, W. P. (1995). Opiate mechanisms in self-injury. Mental Retardation and Developmental Disabilities Research Reviews, Special Issue: Self-injury in developmental disabilities: Neurobiological and environmental mechanisms, 1(2), 130-136. https://doi.org/10.1002/mrdd.1410010209

Sher, L., \& Stanley, B. (2009). Biological models of nonsuicidal self-injury. In M. K. Nock (Ed.), Understanding nonsuicidal self-injury: Origins, assessment, and treatment (pp. 99-116). Washington, DC: American Psychological Association. https://doi.org/10.1037/11875-006

Simeon, D., Stanley, B., Frances, A., Mann, J. J., Winchel, R., \& Stanley, M. (1992). Self-mutilation in personality disorders: Psychological and biological correlates. American Journal of Psychiatry, 149(2), 221-226. https://doi.org/10.1176/ajp.149.2.221

Walsh, B. W. (2006). Treating self-injury: A practical guide (pp. 74-77). New York: Guilford Press.

Weierich, M. R., \& Nock, M. K. (2008). Posttraumatic stress symptoms mediate the relation between childhood sexual abuse and nonsuicidal self-injury. Journal of Consulting and Clinical Psychology, 76(1), 39-44. https://doi.org/10.1037/0022-006X.76.1.39

Whitlock, J., Eckenrode, J., \& Silverman, D. (2006). Self-injurious behaviors in a college population. Pediatrics, 117(6), 1939-1948. https://doi.org/10.1542/peds.2005-2543

Xiao, Y. N. (2007). Research on childhood abuse and adolescent self-injurious behavior. Master's Thesis, Anhui medical university.

Yates, T. M., Carlson, E. A., \& Egeland, B. (2008). A prospective study of child maltreatment and self-injurious behavior in a community sample. Development and Psychopathology, 20(2), 651-671. https://doi.org/10.1017/S0954579408000321

Yu, L. X., Jiang, G. R., \& Wu, C. Z. (2011). Psychological evaluation and treatment of nssi. Chinese Journal of Mental Health, 25(12), 937-941.

Yu, L. X., Ling, X., \& Jiang, G. R. (2013). Impulsivity of nssi in adolescents. Acta Psychologica Sinica, 45(3), 320-335. https://doi.org/10.3724/SP.J.1041.2013.00320

Zheng, D. D., Bi, X. J., \& Liu, L. F. (2019). Research progress of non-suicidal self-injure behaviors in patients with depression. Journal of Psychiatry, 32(2).

Zheng, Y. (2006). Investigation on the prevalence of self-injury behavior among middle school students in wuhan and its functional model. Master Thesis, Central China normal university.

Zhou, L., He, X. Y., \& Xiao, S. Y. (2006). Methodological problems of impulse measurement. Chinese Journal of Clinical Psychology, 14(5), 455-457.

\section{Copyrights}

Copyright for this article is retained by the author(s), with first publication rights granted to the journal.

This is an open-access article distributed under the terms and conditions of the Creative Commons Attribution license (http://creativecommons.org/licenses/by/4.0/). 